Article

\title{
One method towards the trisection of the angle
}

\section{Gerasimos T. Soldatos}

Pindarou 43, 56224 Thessaloniki, Greece.

Taburishanska 73, 27505 Svetlovodsk, Ukraine.; soldgera@yahoo.com

Received: 17 December 2019; Accepted: 17 January 2019; Published: 22 February 2020.

Abstract: This article maintains that the impossibility of trisection is based on a cubic polynomial whose trigonometric content is not clear; or, the impossibility may be referring to one particular trisection method even if the cubic equation does constitute the equation of trisection. It next proceeds to trisection "indirectly" by attempting to construct one of the two trisectors on the basis of reductio ad absurdum.

Keywords: Trisection of acute angle, Vieta's polynomials, trisection equation.

MSC: 51M15, 11C08, 03F03.

\section{Introduction}

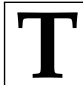

he Trisection of an arbitrary acute angle by means of a straightedge and a compass was deemed by the ancient Greeks to be impossible. In Book IV of his "Mathematical Collections", Pappus of Alexandria (c. 290-c. 350) writes: "Ěgeometers of the past who sought by planes to solve the Ě problem of the trisection of an angle, which is by its nature a solid problem, were unable to succeed. For they were as yet unfamiliar with the conic sections and were baffled for that reason. But later with the help of the conics they trisected the angle using the following 'vergings' for the solution..." [1, p. 146].

In 1837, Pierre Laurent Wantzel (1814-1848, [2]) "proved" the impossibility formally. From the triple-angle formulas of Trigonometry, we know that, for angle $\omega$ that;

$$
\tan ^{3} \omega-3 \tan 3 \omega \tan ^{2} \omega-3 \tan \omega+\tan 3 \omega=0 .
$$

This, equation is supposed to be an irreducible polynomial equation, $x^{3}-3 A x^{2}-3 x+A=0$, and cubic roots are not geometrically constructible. Yet, from Franciscus Vieta's (1540-1603) recurrence formulas, we have $\tan (v+1) \omega=[\tan (v \omega)+\tan \omega] /[1-\tan (v \omega) \tan \omega]$, or letting $\tan (v+1) \omega=\alpha$ and $\tan \omega=x$, and using the recurrence formula for $\tan (v \omega)$,

$$
\alpha-x \alpha \frac{\tan (v-1) \omega+x}{1-x \tan (v-1) \omega}-\frac{\tan (v-1) \omega+x}{1-x \tan (v-1) \omega}-x=0,
$$

and using again the recurrence formula for $\tan (v-1) \omega$,

$$
\alpha-x \alpha \frac{\frac{\tan (v-2) \omega+x}{1-x \tan (v-2) \omega}+x}{1-\frac{\tan (v-2) \omega+x}{1-x \tan (v-2) \omega} x}-\frac{\frac{\tan (v-2) \omega+x}{1-x \tan (v-2) \omega}+x}{1-\frac{\tan (v-2) \omega+x}{1-x \tan (v-2) \omega} x}-x=0,
$$

or letting $\tan (v-2) \omega \equiv y$, and after some operations,

$$
x^{3}-3 \frac{\alpha-y}{1+\alpha y} x^{2}-3 x+\frac{\alpha-y}{1+\alpha y}=0 .
$$

This cubic equation is again of the form of the trisection polynomial: $x^{3}-3 A x^{2}-3 x+A=0$. But, it is neither an equation for $v$-section, because one should have $x^{v}$ rather than $x^{3}$, nor an equation for the trisection of an angle equal to $3 \omega$, because then $(\alpha-y) /(1+\alpha y)=\alpha \Rightarrow y\left(\alpha^{2}+1\right)=0$ and hence, that either $y=0$ or $\alpha^{2}=-1$, which are both absurd results. Even more absurd would be to let $(\alpha-y) /(1+\alpha y)=A$ in order to make (2) look like what is supposed to be a trisection equation. 
There is clearly a problem on whether the geometric solvability of trisection should be judged on the basis of cubic polynomials like those under (1) or (2). To complicate the matter even further, note that inserting $v=2$ in $(\alpha-y) /(1+\alpha y)=[\tan (v+1) \omega-\tan (v-1) \omega] /[1-\tan (v+1) \omega \tan (v-1) \omega]$ to get:

$$
\frac{\tan (3 \omega)-\tan \omega}{1-\tan (3 \omega) \tan \omega}=\frac{\alpha-x}{1-\alpha x}
$$

which when in turn is inserted in (2), gives the quartic equation:

$$
\alpha x^{4}+4 x^{3}-6 \alpha x^{2}-4 x+\alpha=0 .
$$

Could (3) be the true equation of trisection? Or, if (1) and (2) are those equations, indeed, does the impossibility refers to one only specific trisection method? In any case, the next section presents a trisection attempt by seeking to construct one of the two trisectors; it does on the basis of reductio ad absurdum.

\section{The Geometry of Trisector}

\section{Problem:}

Trisect a given acute angle $\omega$, with the aid of a straightedge and a compass.

\section{Analysis:}

Suppose that we have trisected angle $\angle \Theta \Omega \Sigma=\omega$ in Figure 1, which also contains the following elements: Bisector $\Omega T$ forms with half-line $\Omega B$, angle $\angle T \Omega B=\epsilon+(\omega / 2)=45^{\circ}$. Also, $\omega_{1}=\omega_{4}=(\omega / 3)$ and $\omega_{2}=$ $\omega_{3}=(\omega / 6)$. The right triangle $\triangle I \Omega \Gamma$, which is formed having hypotenuse the line segment $\Omega I$ of trisector $\Omega O$, is an isosceles triangle as is triangle $\triangle T \Omega B$ so that $\Omega \Gamma=\Gamma I=\Gamma B, \sigma+\tau=90^{\circ}, \sigma=\psi=\tau+v+\chi$, $\zeta=\kappa=\tau=(\omega / 6)$. Given now that $\alpha=\iota=\epsilon=b, 2 b+\omega=90^{\circ}, \varphi+(\omega / 2)=90^{\circ}, \psi+(\omega / 6)=90^{\circ}$, and $\psi=\varphi+(\omega / 3)$, routine calculations of triangle angles yield also the following list of angles:

$\beta=45^{\circ}-(\omega / 6), \gamma=90^{\circ}-b=45^{\circ}+(\omega / 2)=b+\omega, \delta=90^{\circ}+b+(\omega / 6), \eta=45^{\circ}+(\omega / 3)$, $\theta=b+(\omega / 2)=45^{\circ}, \lambda=45^{\circ}+(\omega / 6), \mu=v=\varphi+(\omega / 3), \rho=\omega / 3, t=90^{\circ}-(\omega / 6)=\varphi+(\omega / 3)=s$, $m+n=b+\omega, v+\chi=90^{\circ}-(\omega / 3)$, and $\xi=45^{\circ}+(\omega / 6)=90^{\circ}+b+(\omega / 3)$.

That is, according to this analysis, trisection imposes that $\Sigma O=\Sigma I$. Nevertheless, the Analysis does not determine angles $m, n, v$, and $\chi$. It appears through the sum $m+n=\omega+a$ that $m=\omega$ and $n=\alpha=b$, and this is what will be assumed below.

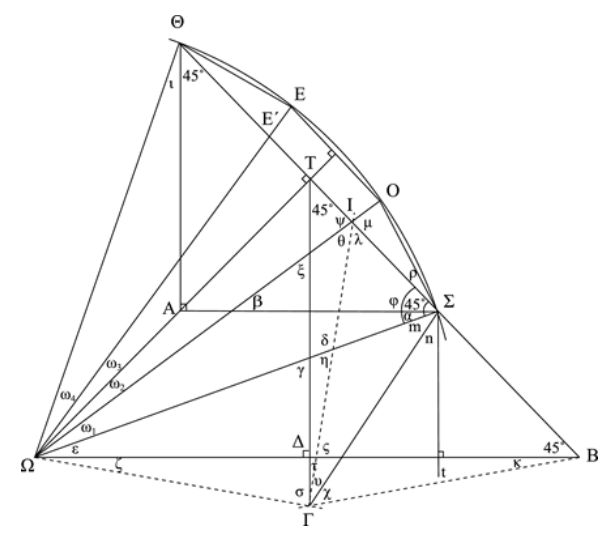

Figure 1. Construction of Trisector

\section{Construction of Trisector $\Omega O(\Omega I)$ :}

Given acute angle $\angle \Theta \Omega \Sigma=\omega$ to trisect, draw bisector $\Omega T$ and form next based on it, angle $\epsilon+(\omega / 2)=$ $45^{\circ}$ and the isosceles right triangles $\triangle \Theta A \Sigma, \triangle T \Omega B$, and $\triangle T \Omega \Delta$. From point $\Sigma$, draw a line parallel to $\Omega \Theta$ and meeting the downward extension of $T \Delta$ at point $\Gamma$. The hypotenuse $\Omega I$ of the isosceles triangle $\triangle \Omega \Gamma I$ formed having side equal to $\Omega \Gamma$, constitutes a segment of the sought trisector $\Omega O$ of $\angle \Theta \Omega \Sigma=\omega$.

Proof (by Contradiction):

In Figure 1, we have by construction, $\Omega \Gamma=\Gamma I=\Gamma B, \sigma+\tau=90^{\circ}, \sigma=\psi=\tau+v+\chi, \zeta=\kappa=\tau=\omega_{2}$, $\alpha=\iota=\epsilon=b, 2 b+\omega=90^{\circ}, \varphi+(\omega / 2)=\varphi+\omega_{1}+\omega_{2}=90^{\circ}, \varphi+\omega_{1}=\psi$, and $\psi+\omega_{2}=90^{\circ}$, where the distinction between $\omega_{1}$ and $\omega_{2}$ has been based on the construction of $\Omega I$. Given these relationships, simple calculations of triangle angles yield all of the angles mentioned in the Analysis, with $\omega_{1}$ and $\omega_{2}$ being now in 
the place of $\omega / 3$ and $\omega / 6$, respectively. I have to show that $\mu=v=\varphi+\omega_{1}=\varphi+\omega_{2}+\left(\omega_{1} / 2\right) \Rightarrow \omega_{1}=2 \omega_{2}$. Suppose that this equality does not hold and that $\Sigma O \Sigma I$. Suppose that some other chord, $\Sigma O^{\prime}$, not $\Sigma O$, is equal to $\Sigma I$. But, then, $\rho \neq \omega_{2}+\left(\omega_{1} / 2\right)$, which would be absurd if that other chord was the one connected with the trisector. The contradiction can be seen through the following metrics, too. Let $d$ denote distance in the $x y$-plane as in Figure 2, where it is assumed that $S O^{\prime}>S O$ :

$$
\begin{aligned}
& d_{S O}=\left[\left(x_{2}-x_{1}\right)^{2}+\left(y_{2}-y_{1}\right)^{2}\right]^{\frac{1}{2}} \\
& d_{S O^{\prime}}=\left[\left(x_{3}-x_{1}\right)^{2}+\left(y_{3}-y_{1}\right)^{2}\right]^{\frac{1}{2}} \\
& d_{O O^{\prime}}=\left[\left(x_{3}-x_{2}\right)^{2}+\left(y_{3}-y_{2}\right)^{2}\right]^{\frac{1}{2}} \\
& d_{S I}=\left[\left(x_{4}-x_{1}\right)^{2}+\left(y_{4}-y_{1}\right)^{2}\right]^{\frac{1}{2}}
\end{aligned}
$$

The inequalities obeyed by $x$ and $y$ need not be observed because differences are squared. Now, if $S O^{\prime}=S I$ or the same, $d_{S O^{\prime}}=d_{S I}$, one obtains that:

$$
\left(x_{3}-x_{4}\right)\left[\left(x_{3}-x_{4}\right)-2 x_{1}\right]-\left(y_{3}-y_{4}\right)\left[2 y_{1}-\left(y_{3}-y_{4}\right)\right]=0
$$

which can be the case when $x_{1}=\left(x_{3}-x_{4}\right) / 2$ and $y_{1}=\left(y_{3}-y_{4}\right) / 2$, given that $x_{3} \neq x_{4}$ and $y_{3} \neq y_{4}$. But, both results are absurd. Similar absurd findings derive when $S O^{\prime}<S O$ is assumed. Hence, it cannot be that $S O^{\prime}>S O$.

Quod Erat Demonstrandum...

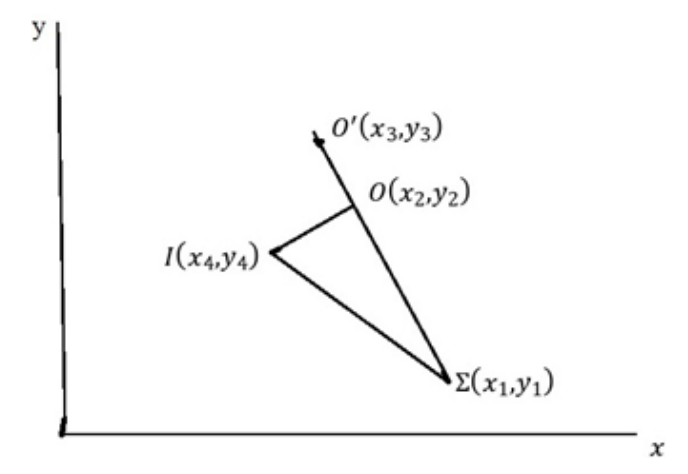

Figure 2. The Metrics of the Contradiction Argument

\section{Epilogue}

The lesson coming out of this article is that there may be geometric constructions deemed impossible on the basis of improper algebra. There have been many geometric trisection attempts through the ages. Suffices to mention those in Forum Geometricorum or in Survey Review and in the literature cited therein. But, the method followed each time leads either to approximate solutions, doing justice to impossibility results, or lacks a methodology, acknowledging Galileo's presumption that "Two truths cannot contradict one another" given the truth of impossibility results. The present essay offers an exact trisection based on a method that does not dispute the truth of these results.

Acknowledgments: This article has benefited from constructive reviewing. Any remaining errors are my own

Conflicts of Interest: "The author declares no conflict of interest."

\section{References}

[1] Pappus, Pappus of Alexandria: Book IV of The Collection. H. Sefrin-Weis, Ed., Berlin: Springer-Verlag 2010.

[2] Wantzel, P.L. (1837). Recherches sur les Moyens de Reconnaître si un Problème de Géométrie peut se Résoudre avec la Règle et le Compas. Journal de Mathématiques Pures et Appliquées, 1, 2, 1837, 366-372. 
(c) 2020 by the author; licensee PSRP, Lahore, Pakistan. This article is an open access article distributed under the terms and conditions of the Creative Commons Attribution (CC-BY) license (http://creativecommons.org/licenses/by/4.0/). 